\title{
Zur Rolle der Dolmetschnotizen beim Konsekutivdolmetschen Ein Erfahrungsbericht
}

\author{
Jana Laukovà (Banská Bystrica)
}

\begin{abstract}
This paper deals with the note-taking system in consecutive interpretation. It discusses the relevance and usefulness of taking notes as well as some essential issues relating to problems which note-taking presents, e.g. whether note-taking can be learnt. If yes, which method is the best one? Symbol for everything or noting words? Which one is the most effective technique? Which language to prefer? The foreign or the target language? Or, another case, is it better to use some international symbols offered by various interpreting schools? The aim of this paper is not to answer those questions, it is rather an attempt to show some famous ideas of interpreters with international recognition like Jean-Francois Rozan, Jean Herbert, Danica Seleskovitch, Heinz Matyssek etc. In spite of their differing views on note-taking, all of them stick to some general principles they recommend: it is important to note the idea, not the word; the interpreter must focus on the main idea; and, at last, notes should be clear, simple and easy to be read.

We can say that each of us, teachers, interpreters and student interpreters, will have our own idea on what note-taking should be. Therefore, it is very difficult to demand the same system of note-taking of all students.

My own experience shows that, at first, students try to note everything which is being said finding out that they can not cope with their notes afterwards. Taking this into account, students should be taught general principles and techniques because they could help students elaborate their own way of note-taking while training their analytical abilities.
\end{abstract}

\section{$1 \quad$ Einführung}

Hinsichtlich der gegenwärtigen Globalisierung, Integration und EU-Erweiterung wird Europa multikulturell, vielsprachig und - insbesondere - dialogbedürftig sein. Es besteht kein Zweifel daran, dass die Sprachen auf dem Weg zum vereinten Europa eine ganz zentrale Rolle spielen und spielen werden müssen. Die Eurobürger sprechen ja verschiedene "Sprachen" nicht nur aus politischer Sicht, sondern auch aus der ethnischen. Damit sie gemeinsame Projekte entwickeln können, müssen sie sich untereinander auch und sogar vor allem auf der elementarsten Ebene verständigen. Der folgende Beitrag, der den Standpunkt einer durchaus technischer Beihilfe an der zwischenmenschlichen Kommunikation ans Tageslicht bringen 
soll, bildet ein kleines Plädoyer für die gemeinschaftstiftende, ja manchmal sehr bedeutsame Rolle der Dolmetscher im Prozess der Kommunikation und Integration.

Der Integrationsprozess vergrössert den Bedarf an fachlich kompetenten Leuten, die diese Kommunikation, vor allem im Fall hochrangiger Verhandlungen in den Bereichen Diplomatie, Politik, Wirtschaft, Gesetzgebung, Verwaltung usw. vermitteln können. Gemeint sind hiermit Dolmetscher und Übersetzer, deren Mangel besonders in den EUKandidatenländern sichtbar ist.

Da ich meinen Erfahrungsbericht (im Prinzip) auf die Problematik des Konsekutivdolmetschens hinorientieren möchte, werde ich mich weiterhin nur zu einigen meines Erachtens nach grundlegenden Aspekten des Dolmetschens und Dolmetscher-Seins äussern. Dies hat aber auch einen anderen Grund: Ich gehe nämlich davon aus, dass lebendiger Dialog und eine ebenso lebendige Kommunikation (gedacht hier sowohl als ein Sinnaustausch wie auch als ein Weg zum Konsens) deutlich erfolgreicher sind als jede andere Art des sozialen Verhaltens, weil sie viel öfter zu Verständigung, Konsens bzw. Kompromiss führen als andere, nicht immer gewalt- und herrschaftsfreie Konfrontationsarten (vgl. dazu: Habermas, Theorie des kommunikativen Handelns, Bd. I-II; Siemek, Vernunft und Intersubjektivität).

\section{Die ersten Ansätze zur Notizentechnik}

Das Konsekutivdolmetschen ist die klassische Form des Dolmetschens im Anschluss an bzw. im Wechsel mit dem Redner. Hier trägt also zunächst der Redner Teile seiner Ausführungen vor, dann übernimmt der Dolmetscher die Übersetzung des jeweiligen Abschnittes in eine Fremdsprache. Dies bedeutet aber, dass der letztere auch die Verantwortung für die Wiedergabe des Aussagesinnes trägt. Allerdings stammt das Konsekutivdolmetschen aus der Zeit, als es noch keine Dolmetscheranlagen gab. Es ist die am besten geeignete Form für formelle und feierliche Anlässe oder Gespräche zwischen Staats- und Regierungschefs. Das Gedächtnis spielt also eine zentrale Rolle bei dieser Art von Dolmetschen und dank der sogenannten Notizentechnik kann der Dolmetscher auch sehr lange Passagen problemlos und rhetorisch gewandt dolmetschen. Demzufolge sind die Dolmetschnotizen eine der Teiloperationen des Konsekutivdolmetschens und dienen als ein Verstehensprotokoll, als eine gewisse Gedächtnisstütze. Deswegen ist ihre Wichtigkeit im Prozess des Konsekutivdolmetschens unbestritten; dies vor allem beim Dolmetschen von längeren Redesegmenten, wo man sich nicht den ganzen Sinn merken kann.

Das Fachdolmetschen gründet auf eine Fähigkeit, die man erst durch systematisches Üben, Training und (selbstverständlich) durch eine langjährige Praxis erreichen kann. In diesem Zusammenhang tauchen dennoch mehrere spezifische Fragen auf, wie folgende: Wie sollte man am effektivsten notieren, mit Hilfe von sogenannten "Symbolen" oder Wortabkürzungen? In welcher Sprache, der Muttersprache oder der Zielsprache? Kann man die Notizentechnik überhaupt erlernen? Laut einer verbreiteten Meinung kann man

"... einen Gedanken sehr wohl genau verstanden haben und ihn damit für einen Augenblick sich als Wissen angeeignet haben. Dennoch besteht die Gefahr, dass man diesen Gedanken in der 
Verdolmetschung auslässt, wenn er nicht durch eine natürliche Gedankenassoziation zum wichtigen Zeitpunkt wieder auftaucht. Und wenn man ihn sich jedoch mit einer entsprechenden Erinnerungshilfe wieder vergegenwärtigt, so kann man ihn mühelos entwickeln. Dies ist die Funktion der Dolmetschnotizen" (Kapp 1984, 99).

Dementsprechend sind die Notizen kein Stenogramm des Gehörten, wie viele denken könnten. Der unverarbeitete Wortlaut wird beim Konsekutivdolmetschen zwar nicht (einfach) so notiert, wie der Dolmetscher ihn hört; es geht eher darum, den von den Wörtern des Redners getragenen Sinn (womöglich) schnell zu erfassen, zu analysieren und in zweckmäßiger Weise auf Papier zu bringen (vgl. Kautz 2000, 313). Und das ist keineswegs einfach. Die wenig erfahrenen Dolmetscher scheinen es zu vergessen, dass nicht das Wort bzw. der Ausdruck, sondern vielmehr dessen Sinn von entscheidender Bedeutung ist.

Die Notizen sind das Ergebnis der Analyse des Inhalts, nur ein mnemotechnisches (gedächtnisstützendes) Mittel zur Vergegenwärtigung dessen, was der Dolmetscher beim Hören verstanden und verarbeitet hat. Sie können jedoch ohne (treffsicheres) Verstehen auf Dauer nicht funktionieren.

Was die ersten Ansätze auf dem Gebiet der Notizentechnik betrifft, lassen sich im Wesentlichen mehrere Grundrichtungen oder "Schulen" erkennen, die die Entwicklung der Notizentechnik beeinflusst haben. Vor allem folgende Personen haben diese Schulen massgeblich mitgestaltet: Jean-Francois Rozan und Jean Herbert, Vertreter der Genfer Schule; weiterhin Danica Seleskovitch, Vertreterin der Pariser Schule und Heinz Matyssek, Verterter der Heidelberger Schule. Zu erwähnen in diesem Zusammenhang sind aber auch drei russischsprachige Arbeiten von R.K. Minjar-Beloru'cev, die bereits 1959 und 1969 zum Thema "Konsekutivdolmetschen" in Moskau herausgekommen sind und auch anregende Darlegungen zur Notizentechnik enthalten.

Die Ansichten dieser Dolmetschtheoretiker und -praktiker über den möglichen Inhalt, bzw. die Didaktik einer Notationslehre sind unterschiedlich und gehen (meistens) auseinander. Es besteht z.B. Uneinigkeit darüber, ob eine Notationslehre überhaupt erforderlich ist. Und wenn es schon der Fall ist, wann Notizen gelehrt werden sollen, ob und inwieweit Symbole zu benutzen sind, wie abgekürzt werden soll usw. (vgl. Kalina 2002, 211).

Rozan, der in den 50er Jahren die ersten Ansätze für eine systematische Notationsdidaktik entwickelt hat, hält die Notizen für eine unentbehrliche Gedächtnisstütze und geht davon aus, daß es allgemein gültige Grundprinzipien gibt, die auch unbedingt lehrbar seien. Er selbst befürwortet ein klares Abkürzungssystem für Wörter, durch Schreiben des Wortanfangs und Hochstellung der letzten Buchstaben des Wortes. Ebensowohl plädiert er für eine zielsprachenorientierte Notation, wenige Symbole und eine klare vertikale Struktur der Notizen.

Danica Seleskovitch geht wiederum davon aus, dass gar nicht die Wörter, sondern allein der den Wörtern entnommene Sinn notiert werden kann (und soll). Die Notizen seien durchaus hochindividuell und nicht vermittelbar. Die Autorin sieht die Notation als reine Memorierungsstrategie für den Notierenden und die Notation ist damit auch nur für den 
Augenblick konzipiert, also nicht geeignet, langfristig Inhalte zu reproduzieren (vgl. Nováková 1993, 13f). Seleskovitch neigt demnach zur zielsprachenorientierten Notation, die auf Verstehen beruht.

Dagegen postuliert der Vertreter der Heidelberger Schule Heinz Matyssek eine sogenannte sprachlose, auf Symbolen beruhende Notation. Das Notieren in Symbolen dient seiner Meinung nach einer (relativ) freien Wiedergabe des Textes in der Zielsprache. Symbole sieht er als effiziente und knappe Notationselemente. Unter "Symbol" versteht er nicht nur das von jedem sprachlichen Bezug und jeder sprachlichen Erscheinungsform gelöste Zeichensymbol, sondern "jedes Notationszeichen, das in dieser oder jener Form als "Sinnträger", als "Bedeutungsverkörperer" auftritt (vgl. dazu Matyssek 1989, 157).

In Matyssek's Handbuch der Notizentechnik für Dolmetscher, Teil I kann man ausser einer theoretischen Grundlegung eine sehr ausführliche Auflistung von Symbolen finden, wobei sie nach verschiedenen Teilgebieten (z.B. Arbeit, Wirtschaft, Politik usw.) unterteilt sind. Es gibt spezielle Symbole (Zeichen, Wortabkürzungen) für mehrere Wortgruppen. Zudem verzeichnet er im Teil II einige alphabethische Listen der meistgebrauchten Wörter, die mit Hilfe von Symbolen notiert werden können. Darüber hinaus ist Heinz Matyssek davon überzeugt, dass eine zielsprachenorientierte Notation nur dann möglich ist, wenn die Zielsprache die Muttersprache ist.

Alle an dieser Stelle nur kurz erwähnten Meinungen in Bezug auf das Notizensystem haben etwas Gemeinsames, und zwar einen gemeinsamen Nenner in den Aussagen über Notation: Effizienz, Knappheit und Unverwechselbarkeit der Notizen. Dies sind die Grundprinzipien, die von den verschiedenen Autoren immer wieder als sinnvoll erachtet werden (vgl. Kalina 2002, 212). Ebenso sind sich die meisten Theoretiker und Praktiker einig, dass die Notizentechnik eindeutig mit in die Ausbildung zum Dolmetscher gehört.

\section{Die Form der Notizen}

Die Notizentechnik soll vor allem einfach, ökonomisch, klar und schnell erfassbar sein. Ausser diesen Merkmalen sind hier die logischen Verbindungen zwischen den einzelnen Sinnschritten der Darlegungen von entscheidender Bedeutung.

Die einfachste Form von Notizen ist bei den meisten Dolmetschern irgendein Wort der Aussage (beispielsweise: Präsident = Präs.), ausgeschrieben oder abgekürzt, oder ein "Symbol", wie z.B. ein Zeichen aus der Mathematik, ein griechischer Buchstabe, ein Autokennzeichen (SK, A.) als Bezeichnung der Länder, eine stilisierte Zeichnung (z.B. eine Menschenfigur als Symbol für eine Person; ein Stuhl als Symbol für Sitzung, Tagung usw.). Der Phantasie sind keine Grenzen gesetzt, wichtig ist nur, dass jeder Dolmetscher für sich mit Hilfe dieser Notizen nachher eine befriedigende Wiedergabe zu Stande bringt und den Sinn der Aussage reproduzieren kann. Viele dieser Notationselemente sind sprachenunabhängig, d.h. übereinzelsprachlich.

Der Dolmetscher notiert ausführlicher und wörtlich, was er nicht näher gedanklich analysieren kann, wie z.B. Zahlen, Eigennamen usw. Die Symbole müssen wie jede andere 
Schrift mühelos gelesen werden können, sonst werden sie eher zu einem Hindernis. Deshalb geht allerdings die grosse Mehrzahl der Dolmetscher damit sparsam um (vgl. Kapp 1984, 101).

Für manche Dolmetscher ist es dennoch nicht empfehlenswert, eine zu grosse Anzahl von Symbolen zu verwenden, weil sie dann nicht genügend automatisiert werden. Optimal sollen ca. 20-30 permanent verwendete Symbole sein. Dazu kommen noch für eine spezifische Kommunikationssituation ausgedachte Ad-hoc-Symbole (z.B. ein Friedenszeichen als Symbol des Kompromisses usw.) (vgl. Kautz 2000, 319).

Die Ausführlichkeit des Notierens hängt von der jeweiligen indivuduellen Konzentrationsfähigkeit des Dolmetschers und dem behandelten Thema ab. Es genügt oft, ein einziges Wort zu notieren, mit dessen Hilfe der Dolmetscher dann den verstandenen gedanklichen Inhalt bei der Wiedergabe frei entwickeln kann. Viele Dolmetschpraktiker postulieren eine vertikale Gliederung der Informationen im Notat. Diese wird mit dem stufenförmigen horizontalen Einrücken der Abstufung kombiniert, d.h. wesentliche Satzglieder haben im Notat einen festen Platz: z.B. Subjektgruppe ganz links, etwas darunter und mehr rechts das Prädikat, etwas darunter und wieder mehr rechts das Objekt usw. (vgl. hierzu Kautz 2000, 316). Solche Art der Notation führt zu grösserer Überschaubarkeit und Klarheit.

Ansonsten überwiegt die Ansicht, dass die Anfänger mehr in der Ausgangssprache und Fortgeschrittene mehr in der Zielsprache notieren.

\section{$4 \quad$ Abschlusswort}

Die Notizentechnik gehört eindeutig zur Ausbildung zum Konsekutivdolmetscher, weil sie, wie schon am Anfang erwähnt, eine der Teiloperationen des Konsekutivdolmetschens ist und mit ihrer Hilfe kann der Dolmetscher bei längeren Passagen den Redesinn möglichst effektiv zu Stande bringen.

Nun ein kurzes Beispiel aus meiner eigenen Praxis: Am Anfang versuchen die Studenten in meinen Lehrveranstaltungen im Fach Konsekutivdolmetschen alles mitzuschreiben, was sie hören. Sie stellen aber nach kurzer Zeit selbst fest, dass es ihnen die Arbeit wesentlich erschwert, weil sie sich mehr auf das Schreiben konzentrieren als auf den Textsinn. Meistens versuchen sie sogar ganze Wörter mitzuschreiben, was viel Zeit in Anspruch nimmt. Infolgedessen können sie die Wiedergabe des Textes in der Zielsprache nicht zu Stande bringen. Später bekommen sie Listen mit schon existierenden und von Dolmetschpraktikern verwendeten Symbolen (z.B. Matyssek: Handbuch der Notizentechnik für Dolmetscher Teil III). Diese Symbole sollen sie sich aneignen oder sich selbst für häufig vorkommende Begriffe in den verwendeten Texten eigene Symbole ausdenken (ca. 50 Symbole oder Zeichen). Die betroffene Studentengruppe geht systematisch vor und analysiert gründlich alle Schritte der Notation. Es wird Nachdruck gelegt auf das Notieren der Schlüsselwörter und die Kompression (siehe Bohusova 2002, 120). Zu ersten praktischen Übungen gehören z.B. 
Vortrag einer kurzen Rede, Pausen für die Notizennahme nach den einzelnen Sinnschritten, danach Verdolmetschung, Besprechung der Notizen und der Verdolmetschung usw.

Daraus ergibt sich allerdings, dass sich jeder Dolmetscher selbst ein ihm am besten angepasstes System von Notizen erarbeiten, also seine eigene Technik des Notierens entwickeln muss, das heisst eine Kombination von Abkürzungen, Zeichen sowie Symbolen, die für ihn spezifisch und optimal sind.

Man kann nicht pauschal verallgemeinern, dass es reicht, einfach diese Symbole oder Zeichen auswendig zu lernen, um ein erfolgreicher Dolmetscher zu werden. Wie bereits am Anfang erwähnt, sind die Notizen eine der grundlegenden Teiloperationen des Konsekutivdolmetschens. Das ganze Verfahren bildet dann einen ganzheitlichen, synthetischen Prozess, der aus mehreren Teiloperationen besteht. Und nur unter der Bedingung, dass man alle diese Operationen erfolgreich durchgeführt hat, kann die Qualität der Dolmetschleistung perfekt sein.

\section{Literaturangaben}

Best, Joanna/Kalina, Sylvia (eds.) (2002): Übersetzen und Dolmetschen. Tübingen.

Bohusova, Zuzana (2002): "Lingvisticke predpoklady fonetickych recovokomunikacnych a tlmocnickych stereotypov". In: Preklad a tlmocenie. Zbornik prispevkov z medzinarodnych konferencii v dnoch 5.a 6.juna 2002 v Banskej Bystrici. Banska Bystrica: FiF UMB, Katedra slovakistiky 2002: 115-123.

Habermas, Jürgen (1977): Theorie des kommunikativen Handelns. Frankfurt/M.

Kapp, Volker (ed.) (1984): Übersetzer und Dolmetscher. Tübingen.

Kautz, Ulrich (2000): Handbuch Didaktik des Übersetzens und Dolmetschens. München.

Matyssek, Heinz (1989): Handbuch der Notizentechnik für Dolmetscher. Teil I. Heidelberg.

Nováková, Taida (1993): Tlmočenie. Teória - výučba - prax. Bratislava.

Siemek, Marek (2000): Vernunft und Intersubjektivität. Baden-Baden. 